IUCrJ

ISSN 2052-2525

CHEMISTRY|CRYSTENG

Received 13 February 2017

Accepted 25 October 2017

Edited by V. T. Forsyth, Institut Laue-Langevin, France, and Keele University, UK

Keywords: chirality; structure analysis; configurational change; density-functional-theorybased methods; phase transitions; intermolecular interactions; properties of solids; hydrogen bonding; materials science.

Supporting information: this article has supporting information at www.iucrj.org

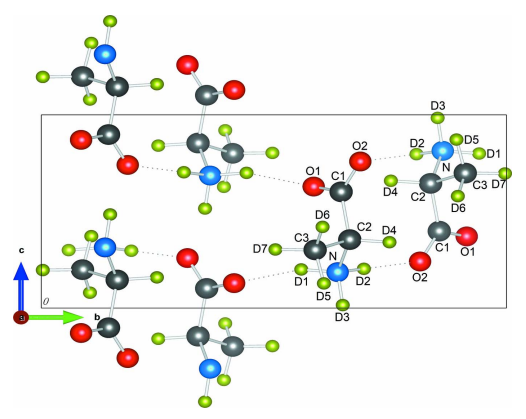

OPEN $\odot$ ACCESS

\section{Hydrogen bonds in crystalline D-alanine: diffraction and spectroscopic evidence for differences between enantiomers}

\author{
Ezequiel A. Belo, ${ }^{a, b}$ Jose E. M. Pereira, ${ }^{c}$ Paulo T. C. Freire, ${ }^{b}$ Dimitri N. Argyriou, ${ }^{d}$ \\ Juergen Eckert ${ }^{\mathrm{e}, \mathrm{f}}$ and Heloisa N. Bordallo ${ }^{\mathrm{c}, \mathrm{e}}{ }^{\mathrm{s}}$
}

a'Faculdade de Física, Universidade Federal do Pará, Belém, Pará, Brazil, 'b Departamento de Física, Universidade Federal
do Ceará, Fortaleza, Ceará, Brazil, ${ }^{\mathbf{C}}$ Niels Bohr Institute, University of Copenhagen, Universitetsparken 5, Copenhagen,
2100, Denmark, ${ }^{\mathbf{d}}$ European Spallation Source, 176, SE-221 00 Lund, Sweden, ${ }^{\mathbf{e}}$ Department of Chemistry, University of
South Florida, 4202 East Fowler Ave, Tampa, FL 33620, USA, and ${ }^{\mathbf{f} T h e o r e t i c a l ~ D i v i s i o n, ~ L o s ~ A l a m o s ~ N a t i o n a l ~ L a b o r a t o r y, ~}$
Los Alamos, NM 87545, USA. *Correspondence e-mail: bordallo@nbi.ku.dk

Enantiomeric amino acids have specific physiological functions in complex biological systems. Systematic studies focusing on the solid-state properties of D-amino acids are, however, still limited. To shed light on this field, structural and spectroscopic studies of D-alanine using neutron powder diffraction, polarized Raman scattering and $a b$ initio calculations of harmonic vibrational frequencies were carried out. Clear changes in the number of vibrational modes are observed as a function of temperature, which can be directly connected to variations of the $\mathrm{N}-\mathrm{D}$ bond lengths. These results reveal dissimilarities in the structural properties of D-alanine compared with L-alanine.

\section{Introduction}

It is well known that chirality plays a fundamental role in the bioactivity of molecules taking part in vital functions of living organisms. Amino acids are fundamental molecules of life and can, except for glycine, be found in enantiomeric $\mathrm{L}^{-}$and D-forms with their chiral centres on the $\alpha$-carbon. Moreover, progress in analytical chemical tools has now established that considerable amounts of D-amino acids are also found in higher mammals including humans (Karakawa et al., 2015). Their distribution and regulation are different from those of the L-forms. It is well known that D-alanine (D-Ala), D-serine (D-Ser) and D-aspartic acid (D-Asp) can be viewed as the main targets for physiological functions and the diagnosis of various diseases, such as chronic kidney disease, endocrine glands disorders, and schizophrenia (Hamase et al., 2002).

Although D-amino acids are now increasingly recognized as physiologically active molecules, as well as potential biomarkers (Kimura et al., 2016), remarkably few studies have been devoted to the understanding of their solid-state properties. As anticipated, most reports do not find any difference in their properties when compared with their chiral counterpart, except in experiments where the chiral character is relevant (Ganesan et al., 2013; Ishikawa et al., 2017). Nevertheless, a number of papers from W. Wang's group (Wang et al., 2000, 2002) have described possible phase transitions in single crystals of D-Ala based on observed differences between crystalline D-Ala and L-alanine (hereafter L-Ala) as a function of temperature. These differences were related to the parity violation energy difference (PVED), which has been searched for in chiral molecules since its conjecture by A. Salam in 1992 (Salam, 1992). However, no evidence for this theory has been 
obtained to date. For instance, Sullivan et al. (2003) have reexamined these measurements, and as well carried out X-ray diffraction and ${ }^{13} \mathrm{C}$ solid-state NMR in both enantiomers of alanine between room temperature and about $250 \mathrm{~K}$, and found no anomalous behaviour. Along these lines Wilson et al. (2005) have further investigated the crystal structures of hydrogenated L-Ala (at 295 and $60 \mathrm{~K}$ ) and D-Ala (at 300, 295, $260,250,240$ and $60 \mathrm{~K}$ ) using single-crystal neutron diffraction. Once more, no clear structural changes were found, which could have supported the anomalies observed in the bulk measurements, and in turn be indicative of the observable effect of PVED.

Careful scrutiny of the results reported in Wilson et al. (2005), however, led us to realise that changes in the $\mathrm{N}-\mathrm{H}$ covalent bond distances as a function of temperature in D-Ala appeared to be different from those observed for L-Ala. The data for fully hydrogenated single-crystal results from Wilson et al. (2005) are given as supplementary information (Fig. S1). Data obtained by Lehmann et al. (1972) and Destro et al. (2008) for L-Ala are also reported. These findings as well as the biological relevance of D-Ala gave rise to the investigations reported in this paper. Here the structural stability of fully deuterated D-Ala was analysed by means of neutron powder diffraction (NPD), while the dynamics of hydrogenated D-Ala were analysed using single-crystal polarized Raman spectroscopy (RS).

Herein we report on a number of differences in the dynamical behaviour of D-Ala compared with L-Ala, the most remarkable being the observation of new Raman active modes in the $A$ - and $B$-irreducible representations of the factor group $D_{2}$ below 160 and $260 \mathrm{~K}$, respectively. These findings together with the neutron powder diffraction and density-functionaltheory-based methods (DFT) results show that small differ-

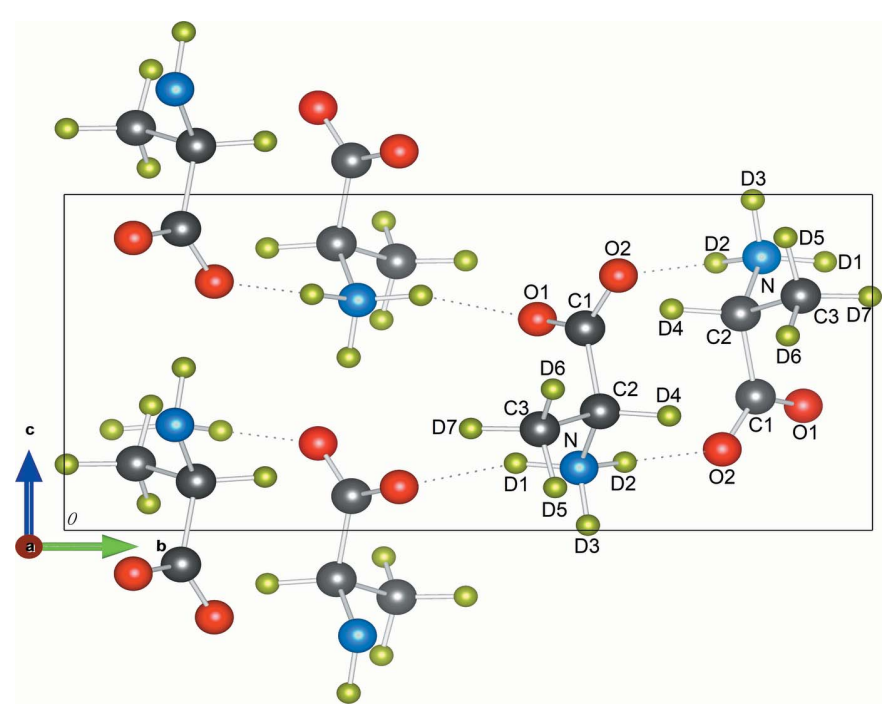

Figure 1

Crystal structure of fully deuterated D-alanine (D-Ala, $\mathrm{C}_{3} \mathrm{D}_{7} \mathrm{NO}$ ) projected onto the $a b$ plane with atoms labelled as in Destro et al. (1988). The $\mathrm{C}$ atoms are shown in grey, $\mathrm{O}$ atoms in red, $\mathrm{N}$ in blue and $\mathrm{D}$ atoms in green. ences in the crystal packing, which were previously disregarded (Wilson et al., 2005), can indeed induce different rearrangements of the $\mathrm{NH}_{3}{ }^{+}$group in D-Ala and L-Ala. We note that the macroscopic symmetry of the crystal is nevertheless preserved.

\section{Experimental details}

Polarized Raman spectra were collected on hydrogenated $\mathrm{C}_{3} \mathrm{H}_{7} \mathrm{NO}_{2}$ single crystals obtained by a slow evaporation technique from the preparation of saturated solutions using the solubility curve of D-Ala (Dalton \& Schmidt, 1933). Crystals of a few millimetres in size were obtained by this method, powered again and reutilized for preparation of new saturated solutions. This process was repeated three times in order to obtain crystals with higher quality. The crystals were polished and cut into three parallelepipeds with approximately $90 \mathrm{~mm}^{3}$ volume, such that each has $a, b$ and $c$ crystallographic axes perpendicular to the corresponding faces. The $514 \mathrm{~nm}$ line of an argon ion laser operating with an output power of $250 \mathrm{~mW}$ along with a detection system (Horiba T64000 triple spectrometer) coupled to a liquid nitrogen cooling charge-coupled-device (CCD) detector was used to collect the data. The samples were mounted in a cryogenic helium closed-cycle system where the temperature could be varied continuously from $295 \mathrm{~K}$ to $20 \mathrm{~K}$ and maintained constant within $\pm 0.5 \mathrm{~K}$. Six different scattering geometries, $x(y y) x, y(z z) y, z(x x) z, x(y z) x, y(x z) y, z(x y) z$, were analysed in the backscattering geometry.

Neutron powder diffraction (NPD) measurements on the fully deuterated D-Ala, $\mathrm{C}_{3} \mathrm{D}_{7} \mathrm{NO}_{2}$, purchased from Cambridge Isotope Laboratories and used without further treatment, were performed on the D2B diffractometer located at the ILL. Data were collected at a wavelength of $1.594 \AA$ in small temperature intervals between 4 and $280 \mathrm{~K}$. This instrument is well suited for an accurate determination of lattice constants, the internal atomic coordinates and a refinement of crystal structure with high resolution. The temperature-dependent powder diffraction data were analysed using the crystallographic model of Destro et al. (1988) as initial input with the GSAS suite of programs (Larson \& Von Dreele, 1994). The labelling scheme of the atoms is shown in Fig. 1. Atomic positions were refined together with lattice constants, isotropic atomic displacement parameters and instrumental peak shape parameters. Special attention was paid to modelling of the background, as the thermal diffuse scattering contribution is significant.

Two types of $a b$ initio calculations of harmonic vibrational frequencies were carried out to assist with the analysis of the vibrational spectra. The Raman spectrum was calculated for the isolated molecule using Gaussian09 (Frisch et al., 2009), using as reference the atomic coordinates of the D-Ala at $280 \mathrm{~K}$. The structure was optimized using the polarized continuum model (PCM) of the self-consistent reaction field (SCRF) theory together with the DFT B3LYP level of theory using a $6-311++G(d, p)$ basis set. Vibrational frequencies of crystalline D-Ala were obtained by periodic calculations on a 
$2 \times 1 \times 2$ supercell of the alanine crystals with the Vienna Ab initio Simulation Package (VASP) (Kresse \& Furthmüller, 1996), using the Perdew-Burke-Ernzerhof (PBE) functional along with Vanderbilt ultrasoft pseudopotentials (Perdew et al., 1996) with a plane wave kinetic energy cutoff of $450 \mathrm{meV}$. A $4 \times 4 \times 4$ Monkhorst-Pack mesh of $k$ points (Monkhorst \& Pack, 1976) was used to further improve agreement at lower frequencies. This methodology was first applied to optimize the positions of the atoms within the $2 \times 1 \times 2$ supercell. The optimized atomic positions were in turn used to calculate the harmonic frequencies and atomic vibrational amplitudes in the enantiomers for computing the INS spectra, including folding with the experimental resolution function using the program aClimax (Ramirez-Cuesta, 2004).

\section{Results and discussion}

3.1. Raman scattering: anomalies in the lattice modes of hydrogenated D-Ala

In this section we will discuss the Raman spectra of D-Ala between 30 and $180 \mathrm{~cm}^{-1}$ for six different scattering geometries $[z(x x) z, x(y y) x, y(z z) y, x(y z) x, y(x z) y, z(x y) z]$ and between 200 and $600 \mathrm{~cm}^{-1}$ in the $x(y y) x, y(z z) y$ geometries as a function of temperature. Frequency changes in the low spectral region will give insight into deformation of the crystalline lattice, while in the medium region of the spectrum we can follow the evolution of the $\mathrm{NH}_{3}{ }^{+}$group, which in L-Ala appears at around $480 \mathrm{~cm}^{-1}$ (Susi \& Byler, 1980; Bordallo et al., 1997; Zhang et al., 2015).

According to a group theory analysis, the D-Ala crystal possesses 153 optical modes divided into the irreducible representations of $D_{2}$ factor group as $39 A+38 B_{1}+38 B_{2}+38$ $B_{3}$ of which 132 modes are internal modes and 21 modes are external, distributed into 12 librations and 9 translations. Modes observed in the Raman spectra of $z(x x) z, x(y y) x, y(z z) y$
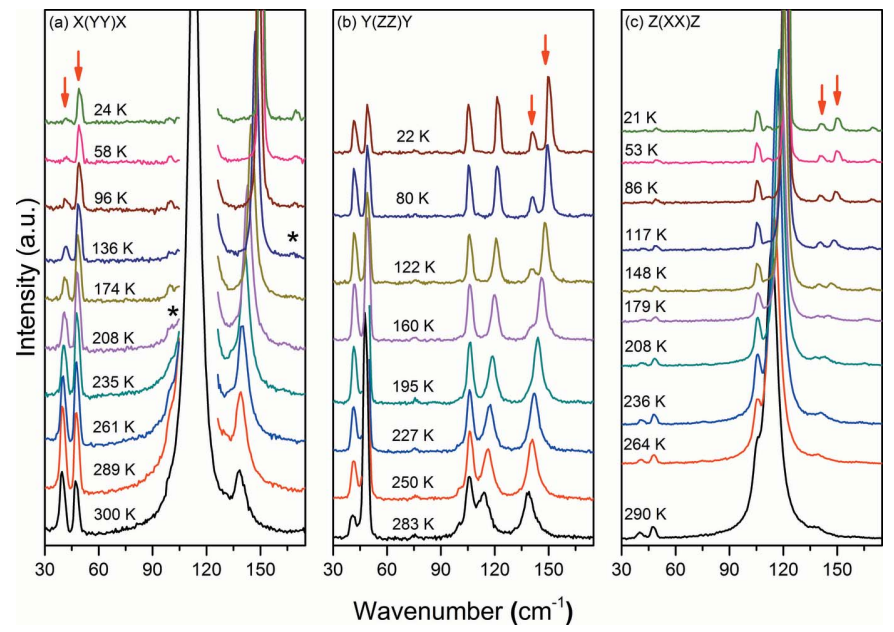

Figure 2

Raman spectra of hydrogenated D-Ala $\left(\mathrm{C}_{3} \mathrm{H}_{7} \mathrm{NO}_{2}\right)$ in the $A$-irreducible representation of the factor group $D_{2}$ for several temperatures between 20 and $300 \mathrm{~K}$ in the region from 30 to $175 \mathrm{~cm}^{-1}$. New bands are marked by $(*)$, while arrows indicate bands that split at lower temperature. scattering geometries are non-polar representing the Raman tensor components $\alpha_{x x}, \alpha_{y y}$ and $\alpha_{z z}$. From the theoretical group analysis, six Raman active modes are expected at low wavenumbers, divided into three translational $(T)$ and three librational $(L)$ modes. $L$-modes, in particular, can be understood as hindered rotations about three perpendicular axes $u$, $v$ and $w$, where $u$ is nearly parallel to the crystal $c$ axis (where a chain of hydrogen bonds links adjacent molecules), $v$ is parallel to the long molecular axis and $w$ is defined as perpendicular to the plane of the molecule (Loh, 1975; Crowell \& Chronister, 1993). The two modes of lowest energy, at $\sim 40$ and $48 \mathrm{~cm}^{-1}$, observed in almost all scattering geometries, are assigned to $w$-axis librations (Loh, 1975; Crowell \& Chronister, 1993).

Initially, we discuss the temperature-dependent Raman spectra of D-Ala for the $x(y y) x$ scattering geometry ( $A$ irreducible representation of the factor group $D_{2}$ ) shown in Fig. 2(a). In this figure it is important to consider the behaviour of the bands at 41 and $48 \mathrm{~cm}^{-1}$, indicated by arrows, which present a notable change of intensity at low temperatures. Interestingly, in the spectrum recorded at room temperature the intensity of the band at $41 \mathrm{~cm}^{-1}$ is greater than the intensity of the band at $48 \mathrm{~cm}^{-1}$, while in the spectrum taken at the lowest temperature, the intensity of the first band is almost zero. It is also possible to observe two small bands, marked by (*), at about 100 and $170 \mathrm{~cm}^{-1}$ below 235 and $175 \mathrm{~K}$, respectively. In spite of their very low intensities, the observation of these L-modes (Loh, 1975; Crowell \& Chronister, 1993) shows a trend in which molecules of D-Ala seem to gain librational degrees of freedom. We point out that both modes belong to the $B$ symmetry, see Fig. 3, and a possible explanation for their appearance is a break of the selection rules due to a subtle phase transition or configurational change in D-Ala. Even if one might consider this observation disputable, and that in reality these new bands are
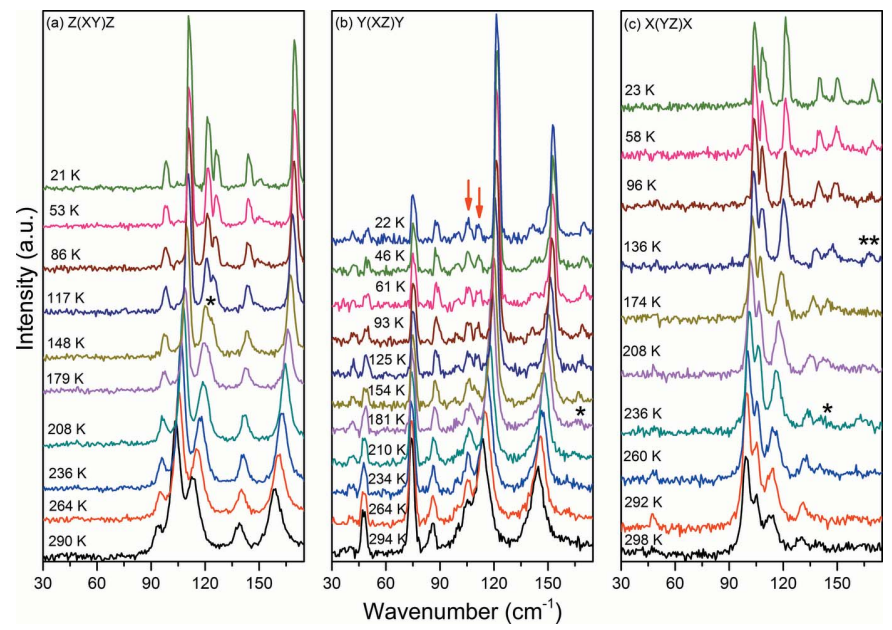

Figure 3

Raman spectra of hydrogenated D-Ala $\left(\mathrm{C}_{3} \mathrm{H}_{7} \mathrm{NO}_{2}\right)$ in the $B$-irreducible representation of the factor group $D_{2}$ for several temperatures between 20 and $300 \mathrm{~K}$ in the region from 30 to $175 \mathrm{~cm}^{-1}$. New bands are marked by $(*)$, while arrows indicate bands that split at lower temperature. 
already present at $300 \mathrm{~K}$, becoming visible on cooling due to their low intensities, other indictations of a configurational change in D-Ala are clear from the RS results.

Let us first consider Fig. 2(b), the spectra of D-Ala recorded in the $y(z z) y$ scattering geometry (also $A$ irreducible representation). In this spectra we observe at $283 \mathrm{~K}$ the presence of a single band located at $\sim 140 \mathrm{~cm}^{-1}$, which clearly splits below $160 \mathrm{~K}$ in two modes located at 140 and $150 \mathrm{~cm}^{-1}$ (marked by arrows) at the lowest temperature. Now we turn to the $z(x x) z$ Raman spectra (also an $A$ irreducible representation of the factor group $D_{2}$ ) depicted in Fig. 2(c). It is clear that the mode at $140 \mathrm{~cm}^{-1}$ also splits into two modes with wavenumbers 140 and $150 \mathrm{~cm}^{-1}$ (marked by arrows) at the lowest temperature.

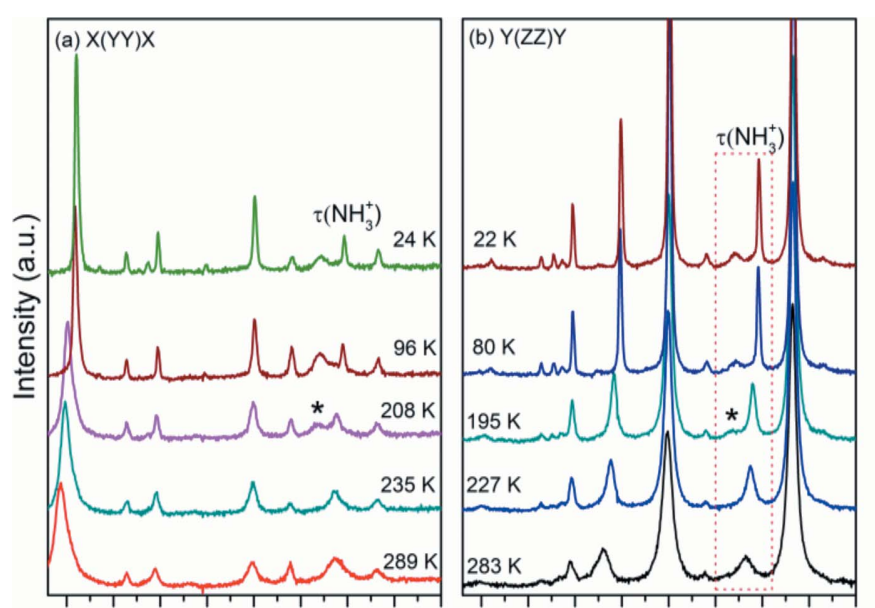

200250300350400450500550600200250300350400450500550600 Wavenumber $\left(\mathrm{cm}^{-1}\right)$

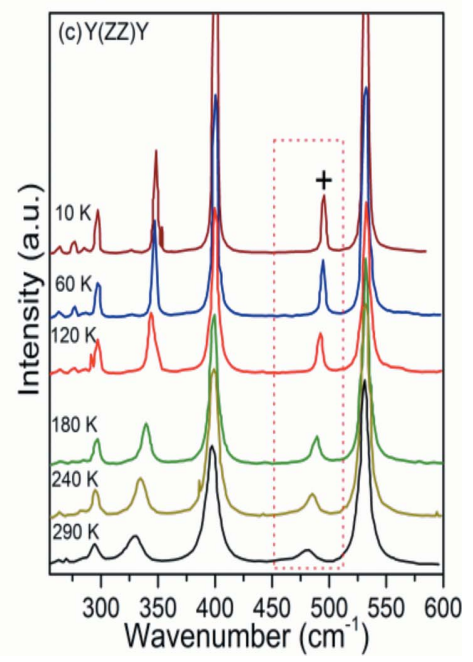

Figure 4

(a) and (b) Raman spectra of hydrogenated D-Ala $\left(\mathrm{C}_{3} \mathrm{H}_{7} \mathrm{NO}_{2}\right)$ in the $A$-irreducible representation of the factor group $D_{2}$ for several temperatures between 20 and $300 \mathrm{~K}$ in the region from 180 to $600 \mathrm{~cm}^{-1}$. A temperature-dependent band at $468 \mathrm{~cm}^{-1}$ appears below $200 \mathrm{~K}$ and is marked by (*). (c) Raman spectra in the $A$-irreducible $y(z z) y$ representation of the factor group $D_{2}$ for selected temperatures between 10 and $290 \mathrm{~K}$ in the region from 250 to $600 \mathrm{~cm}^{-1}$ of hydrogenated L-Ala $\left[\mathrm{C}_{3} \mathrm{H}_{7} \mathrm{NO}_{2}\right.$, adapted from (Vik et al., 2005)]. Note that, differently from D-Ala, the $\tau\left(\mathrm{NH}_{3}{ }^{+}\right)$mode indicated for clarity by $(*)$ in $(b)$, does not split in L-Ala on cooling, as indicated by $(+)$. However, on heating a remarkable wavenumber decrease accompanied by the increase in the linewidth, attributed to the increase in anharmonicity of the torsional vibrations, is observed in both samples.
Finally, the disappearance of the lowest band at $41 \mathrm{~cm}^{-1}$ and the decrease in intensity of the vibration at $48 \mathrm{~cm}^{-1}$ on cooling, also seen in Fig. 2(a), further substantiate the idea that crystalline D-Ala undergoes a structural rearrangement at low temperatures.

Now we turn to the polar modes belonging to the $B$-irreducible representations of the factor group $D_{2}$, Fig. 3. Off diagonal Raman tensor components $\alpha_{x y}, \alpha_{x z}$ and $\alpha_{y z}$ were measured in the following scattering geometries:, $z(x y) z$, $y(x z) y$ and $x(y z) x$. According to a group theory analysis, the modes observed in these configurations are both IR and Raman active. Moreover, due to their scattering geometry, i.e. longitudinal optical phonons that generate a macroscopic electric field producing additional scattering mechanism, it is expected that substantial deformation of the crystalline lattice will be distinctly reflected in their behaviour. At $290 \mathrm{~K}$ five modes, exactly as predicted, were observed in the $z(x y) z$ spectrum, Fig. 3(a), at 93, 103, 113, 138 and $159 \mathrm{~cm}^{-1}$. However below $180 \mathrm{~K}$ the mode at $113 \mathrm{~cm}^{-1}$ splits, giving rise to a new peak marked by $(*)$, and at the lowest temperature the modes are observed at 121 and $126 \mathrm{~cm}^{-1}$. In Fig. 3(b) we present the $y(x z) y$ spectrum that shows six modes located at $47,74,86,105,113$ and $144 \mathrm{~cm}^{-1}$ at room temperature. Even if in this geometry the signal-to-noise ratio is not top quality, it is possible to observe that the mode located at $105 \mathrm{~cm}^{-1}$ starts splitting below $180 \mathrm{~K}$ and becomes completely separated, 105 and $110 \mathrm{~cm}^{-1}$, at the lowest temperatures (marked by arrows). In addition, an extremely weak mode at $165 \mathrm{~cm}^{-1}$, marked by $(*)$, can be observed below $210 \mathrm{~K}$. Finally, in Fig. 3(c) the $x(y z) x$ Raman spectrum is presented, showing five modes at $99,105,114,131$ and $138 \mathrm{~cm}^{-1}$. On cooling two new modes are observed. One is seen around $260 \mathrm{~K}$ [marked by $(*)$ ] and the other around $136 \mathrm{~K}$ [marked by $(* *)]$ located at 150 and $169 \mathrm{~cm}^{-1}$, respectively, at the lowest temperature. Additionally, an inversion of the intensities of the modes located at 99 , 105 and $114 \mathrm{~cm}^{-1}$ occurs. The mode at $47 \mathrm{~cm}^{-1}$, which completely disappears on cooling, is most likely a leak of polarization due to imperfections on the crystal faces.

In the spectral range between 180 and $600 \mathrm{~cm}^{-1}$ (Fig. 4), we can observe four strong bands at $300,400,496$ and $532 \mathrm{~cm}^{-1}$ (at $22 \mathrm{~K}$ ) in the $y(z z) y$ configuration, which are attributed to the $\mathrm{CH}_{3}$ torsion, skeletal rocking, $\mathrm{NH}_{3}{ }^{+}$torsion (Wang \& Storms, 1971; Barthès et al., 2002; Kolesov \& Boldyreva, 2011; Zhang et al., 2015) [labelled in Fig. $4(b)$ as $\tau\left(\mathrm{NH}_{3}{ }^{+}\right)$], and to a mix of intermolecular vibrations. Of more interest, however, is the appearance of a mode at $468 \mathrm{~cm}^{-1}$ below $220 \mathrm{~K}$ [marked by $(*)$ ], distinctively absent in the periodic DFT calculations, which find no mode at all in this region down to $420 \mathrm{~cm}^{-1}$ [Fig. 5(a), top lines] as well as in the Raman spectra of L-Ala (Vik et al., 2005), see Fig. 4(c). This vibration is, however, observed in L-alanine aluminium nitrate, LAAN (Hudson et al., 2009) at $454 \mathrm{~cm}^{-1}$. In LAAN this unassigned vibration has an intensity roughly identical to that of the $\tau\left(\mathrm{NH}_{3}{ }^{+}\right)$and is separated by approximately $30 \mathrm{~cm}^{-1}$ from the $\tau\left(\mathrm{NH}_{3}{ }^{+}\right)$mode (Barthès et al., 2002; Lagaron, 2002), therefore its nature was related to an apparent structural change involving motion of a proton at low temperature. 
A most noteworthy difference in the low-frequency dynamics of $\mathrm{D}$ - and $\mathrm{L}$-alanine is apparent when comparing the calculated $10 \mathrm{~K}$ INS spectra for D-Ala versus $\mathrm{L}$-Ala, Fig. 5(b). It is quite obvious that there are significant differences in the vibrational amplitudes (i.e. peak intensities) of the lowfrequency modes below $350 \mathrm{~cm}^{-1}$, while the high-frequency portion of the INS spectra for the two crystals are very similar. This result can be considered as a further indication that the intermolecular interactions in L- and D-Ala differ because the local symmetry of the enantiomers is not identical. In the VASP minimized structure, the positions of the atoms not involved in the chirality were found to be the same, while the three hydrogen-bond geometries differ between the enantiomers and were found to be similar to those reported in (Wilson et al., 2005), see Table $\mathrm{S} 1$ in the supplementary information. We can, therefore, hypothesize on the basis of all these observations that in crystalline D-Ala a rearrangement of the hydrogen bonds, and in particular a change in the displacement potential for the $\mathrm{NH}_{3}{ }^{+}$protons, may occur which leads to breaking of the selection rules by lowering the local symmetry. In order to better evaluate these spectral anomalies we now turn to the analysis of the neutron powder diffraction data.

\subsection{Neutron powder diffraction: re-arrangement of the hydrogen bonds in D-Ala}

Analysis of the NPD data has allowed us to precisely measure the evolution of bond lengths in D- and L-Ala as a function of temperature and draw correlations between their evolution and changes in the Raman data. The advantage of this approach is the self consistency in the data with respect to systematic errors that reveals this evolution of bond lengths as

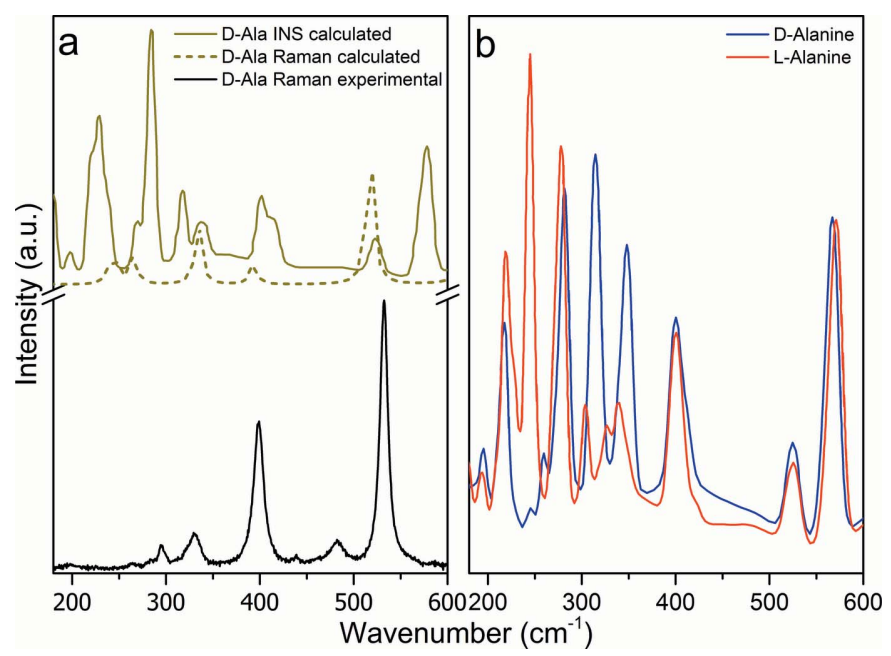

Figure 5

(a) Calculated and experimental Raman spectra of D-Ala $\left(\mathrm{C}_{3} \mathrm{D}_{7} \mathrm{NO}_{2}\right.$ and $\mathrm{C}_{3} \mathrm{H}_{7} \mathrm{NO}_{2}$, respectively) between 150 and $600 \mathrm{~cm}^{-1}$ at $280 \mathrm{~K}$. Comparison is also made with the calculated INS spectrum at $10 \mathrm{~K}$ for D-Ala $\left(\mathrm{C}_{3} \mathrm{H}_{7} \mathrm{NO}_{2}\right)$ from the periodic DFT calculations. Here we note that in DFT calculations $(0 \mathrm{~K})$ there is no internal mode between $420 \mathrm{~cm}^{-1}$ and $500 \mathrm{~cm}^{-1}$. (b) Comparison of the periodic DFT calculations for fully hydrogenated L- and D-Ala at $10 \mathrm{~K}$ where significant differences in the intensities of some of the peaks, i.e. the attendant vibrational amplitudes, are evident between about $200 \mathrm{~cm}^{-1}$ and about $300 \mathrm{~cm}^{-1}$. opposed to precise comparisons of bond-length distances to past measurements at a limited number of temperatures made using a variety of instruments and radiations (Lehmann et al., 1972; Destro et al., 1988, 2008; Wilson et al., 2005). Regardless, our results, Fig. 6, are in reasonable agreement with the previously reported single-crystal measurements at $300 \mathrm{~K}$ on hydrogenated D-Ala (Wilson et al., 2005), where the $\mathrm{NH}_{3}{ }^{+}$ group presents two similar $\mathrm{N}-\mathrm{H}$ distances at this temperature, see Fig. S1(b) in the supporting information. We note the agreement of our data with those of Wilson et al. (2005), between 160 and $240 \mathrm{~K}$ where also three different $\mathrm{N}-\mathrm{D}$ distances were observed.

Firstly, turning our attention on the evolution of the N-D bonds as a function of temperature, for both enantiomers, we find a similar behaviour for the $\mathrm{N}-\mathrm{D} 1$ and $\mathrm{N}-\mathrm{D} 3$ bonds. While the $\mathrm{N}-\mathrm{D} 3$ bond, which links the molecules into columns, remains relatively-temperature independent, in contrast we find that the $\mathrm{N}-\mathrm{D} 1$ bond in both cases has the same value at $280 \mathrm{~K}$ and increases on cooling to $175 \mathrm{~K}$, remaining at a relatively constant value below that temperature. The key difference between the $\mathrm{N}-\mathrm{D}$ bonds of $\mathrm{D}-\mathrm{Ala}$ and D-Ala resides in the evolution of the $\mathrm{N}-\mathrm{D} 2$ bond. For DAla, we find that this bond length has a similar value to $\mathrm{N}-\mathrm{D} 3$ at $280 \mathrm{~K}$ and on cooling it decreases in value until $175 \mathrm{~K}$, remaining relatively constant in value on further cooling. We find an opposite behaviour in L-Ala, where $\mathrm{N}-\mathrm{D} 2$ has a similar value to the $\mathrm{N}-\mathrm{D} 1$ bond length at $270 \mathrm{~K}$, while its value increases gradually on cooling. Overall, the lowtemperature behaviour of these bonds in D-Ala and L-Ala is different in that for D-Ala we find that $\mathrm{N}-\mathrm{D} 1, \mathrm{~N}-\mathrm{D} 2$ and $\mathrm{N}-\mathrm{D} 3$ are dissimilar, while for $\mathrm{L}-\mathrm{Ala} \mathrm{N}-\mathrm{D} 1$ and $\mathrm{N}-\mathrm{D} 3$ are somewhat similar and $\mathrm{N}-\mathrm{D} 2$ is smaller in value. These data suggest somewhat different conformations in D- and L-Ala both at high and low temperatures. Careful scrutiny of Fig. S1 will lead to this same conclusion.

Turning our attention to the $\mathrm{D} \cdots \mathrm{O}$ bonds, our measurements also indicate differences in the $\mathrm{D} \cdots \mathrm{O}$ bond lengths for D-Ala and L-Ala. In both enantiomers, we find the temperature dependence of $\mathrm{D}(1) \cdots \mathrm{O}(1)$ and $\mathrm{D}(3) \cdots \mathrm{O}(2)$ to be very similar, both decreasing linearly with temperature. For $\mathrm{D}(1) \cdots \mathrm{O}(1)$, more specifically, the decrease is linear until approximately $100 \mathrm{~K}$, and then this bond length remains relatively constant with further cooling. The most striking difference in the temperature evolution of these bonds is found for the $\mathrm{D}(2) \cdots \mathrm{O}(2)$ bond length. For L-Ala, $\mathrm{D}(2) \cdots \mathrm{O}(2)$ is of similar value and tracks closely the evolution of $\mathrm{D}(1) \cdots \mathrm{O}(1)$, while in sharp contrast the same $\mathrm{D}(2) \cdots \mathrm{O}(2)$ bond in D-Ala shows a much smaller value at $280 \mathrm{~K}$ compared with its, isomer, increases in size on cooling to $160 \mathrm{~K}$, and on further cooling follows a very similar evolution and value of the $\mathrm{D}(1) \cdots \mathrm{O}(1)$ bond length.

The dissimilarities in the temperature evolution of bond lengths that we have identified in the NPD data mirror the differences in the low-frequency Raman modes of L- and DAla, both reflecting conformational differences between the enantiomers. The differences in the higher temperature behaviour of the $\mathrm{D}(2) \cdots \mathrm{O}(2)$ and $\mathrm{N}-\mathrm{D} 2$ bonds, in particular, 
can be directly correlated with the appearance of the new peaks in the Raman data.

In order to understand these results we turn to previous infrared studies performed on isotopically labelled ND-Ala molecules in a hydrogenated L-Ala crystal (Rozenberg et al., 2003), RS studies in fully hydrogenated L-Ala (Kolesov \& Boldyreva, 2011) as well as to more recent studies on the twice methylated amino group of $\mathrm{N}, \mathrm{N}$-dimethylglycine (Kapustin et al., 2014). While Rozenberg et al. (2003) hypothesize that the appearance of the new bands in the spectra of partially deuterated L-Ala reveals an intrinsic hydrogen-bond disorder resulting from different accessible proton positions, the other authors discuss how the $\mathrm{N}-\mathrm{H} \cdots \mathrm{O}$ hydrogen bonds regulate the stability of the main structural unit in crystalline amino acids. Therefore and as a whole, we must consider that while structural methods probe long-range periodic order, RS sensitivity to short-range interactions allows probing heterogeneous hydrogen-bonding systems. Thus, the appearance of the new mode at $468 \mathrm{~cm}^{-1}$ and the band splitting of the $\tau\left(\mathrm{NH}_{3}{ }^{+}\right)$observed in the $\mathrm{RS}$ of $\mathrm{D}$-Ala strongly suggest that the reported structural differences in the two enantiomers are related to dissimilar accessible weakly bounded protons.

\section{Conclusion}

We have investigated the influence of temperature in the structure of D-Ala combining polarized RS, NPD and DFTbased methods. We find that the reorientation of the $\mathrm{NH}_{3}{ }^{+}$ group in D-Ala also induces modification of the $\mathrm{N}-\mathrm{H} \cdots \mathrm{O}$ hydrogen bonds between two neighbouring molecules similarly to L-Ala (Vik et al., 2005). In addition, modes assigned to lattice vibrations (translations and librations of molecules) in the Raman spectrum split on cooling as in L-Ala (Kolesov \& Boldyreva, 2011). What is more interesting and different from L-Ala, is the observation of new Raman active modes in the $A$ and in $B$-irreducible representations for D-Ala below 160 and $260 \mathrm{~K}$, as well as the observation of a temperature-dependent feature at $468 \mathrm{~cm}^{-1}$ below $200 \mathrm{~K}$. The temperature dependence of the Raman spectra and the coincidence of the new feature in the Raman spectra with anomalies in the bond lengths obtained from NPD in the deuterated D-Ala exclude the possibility of inclusions in the sample. Finally, from NPD one observes that the temperature dependence of the $\mathrm{N}-\mathrm{D}$ covalent bonds in D-Ala and L-Ala are quite different in the following way:

(i) For $250 \mathrm{~K}<T<270 \mathrm{~K}$, the $\mathrm{ND}_{3}{ }^{+}$group in L-Ala shows one long $(\mathrm{N}-\mathrm{D} 3)$ and two short $(\mathrm{N}-\mathrm{D} 1$ and $\mathrm{N}-\mathrm{D} 2)$ covalent bonds, in agreement with Lehmann et al. (1972), while DAla has two long $(\mathrm{N}-\mathrm{D} 2$ and $\mathrm{N}-\mathrm{D} 3)$ and one short $(\mathrm{N}-\mathrm{D} 1)$ covalent bond.

(ii) Between $175 \mathrm{~K}<T<250 \mathrm{~K}$ we observe a transition region for both $\mathrm{L}-$ and $\mathrm{D}-\mathrm{Ala}$.

(iii) For $60 \mathrm{~K}<T<175 \mathrm{~K}$, L-Ala shows two long (N-D1 and $\mathrm{N}-\mathrm{D} 3)$ and one short $(\mathrm{N}-\mathrm{D} 2)$ covalent bonds. This is in agreement with Destro et al. (2008) data at $23 \mathrm{~K}$. On the hand,
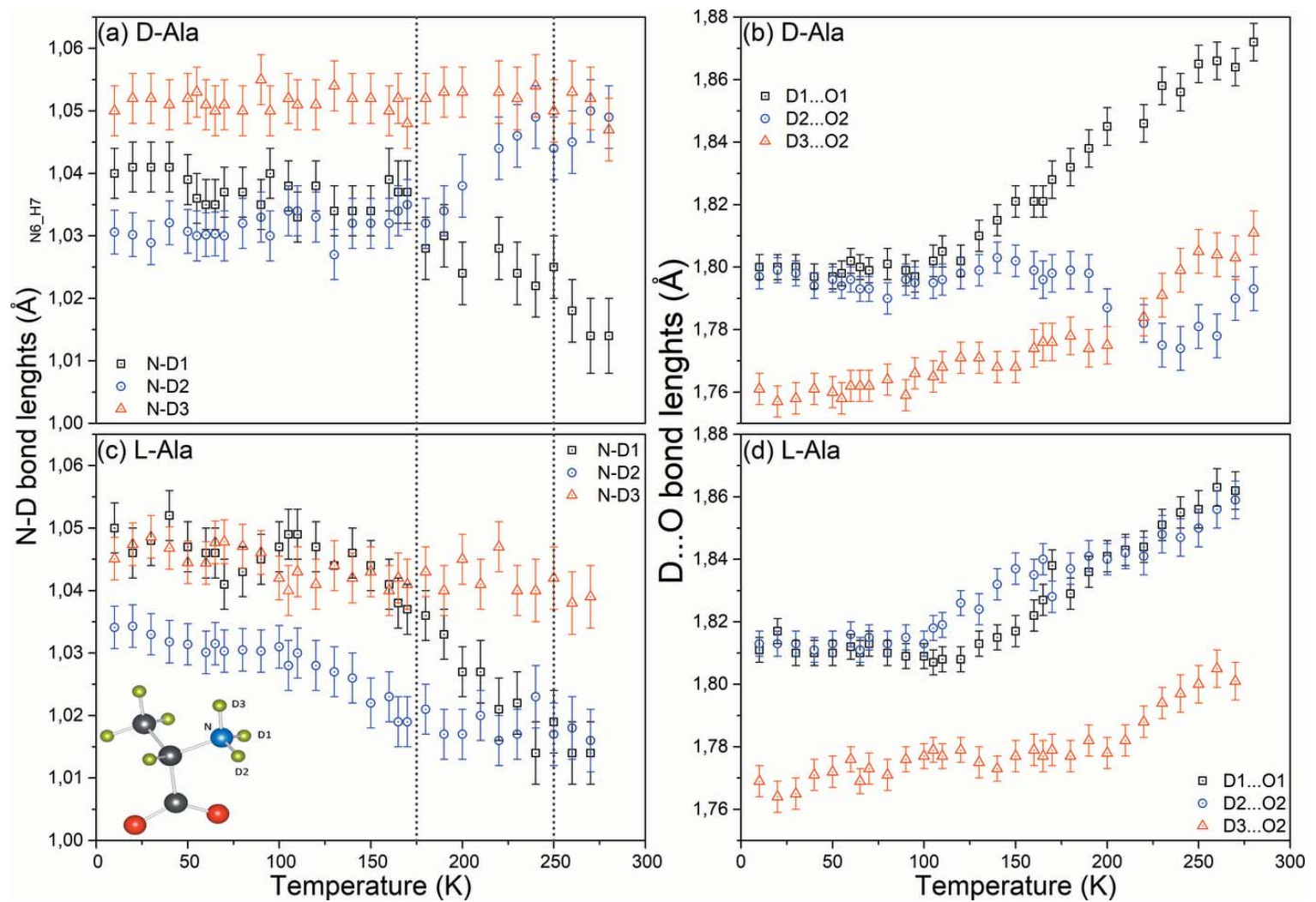

Figure 6

Temperature dependence of the $\mathrm{N}-\mathrm{D}$ and $\mathrm{D} \cdots \mathrm{O}$ bond lengths in D-Ala $\left(\mathrm{C}_{3} \mathrm{D}_{7} \mathrm{NO}_{2}\right)$ are shown in panels $(a)$ and $(b)$. Temperature dependence of the $\mathrm{N}-\mathrm{D}$ and D ... O bond lengths in L-Ala reproduced from De Souza et al. (2007) and recalculated from the data used in De Souza et al. (2009), respectively, are shown in panels $(c)$ and $(d)$. The molecule is represented in the bottom left corner of the figure and labelled in agreement with Destro $e t$ al. (1988). The NPD data were collected on D2B (ILL) using $\lambda=1.594 \AA$. 
and in agreement with Wilson et al. (2005), D-Ala shows two short $(\mathrm{N}-\mathrm{D} 1$ and $\mathrm{N}-\mathrm{D} 2)$ and one long $(\mathrm{N}-\mathrm{D} 3)$ covalent bonds.

(iv) Differently from L-Ala, our data suggest the existence of three dissimilar N-D covalent bonds below $60 \mathrm{~K}$ in D-Ala. This is further supported by the variation in intensity of the vibration located at $41 \mathrm{~cm}^{-1}$ in the $x(y y) x$ representation; this intense peak observed in the spectrum recorded at room temperature basically vanishes on cooling.

Our work therefore leads to the conclusion that even if the crystal symmetry is maintained both L-Ala and D-Ala undergo micro-conformation transitions due to a subtle rearrangement of the hydrogen-bond network (Barthès et al., 2003; Kolesov \& Boldyreva, 2011), which is manifested by the evolution of the bond lengths revealed by NPD and the unexpected RS results. Additionally, a slight difference in crystal packing between the two alanine forms induces distinct dynamics for the hydrogen bonds in D-Ala, which culminates in the observation of extra Raman modes and dissimilar hydrogen-bond arrangements compared with L-Ala.

While D-Ala can be used as a biomarker for kidney disease, the presence of $\mathrm{D}$-Ser is now thought to have an important function in the central nervous system, and D-Asp is reported to regulate the hormonal release in the endocrine glands, no comprehensive studies have been conducted to fully understand the solid-state properties of D-amino acids. Therefore, the results presented in this paper could have important clinical implications, since the reported changes in the hydrogen-bond strength of D-Ala when compared with L-Ala, will cause a direct impact on binding energy, consequently affect its affinity, and lead to disequilibrium between active and inactive conformational receptors (Kržan et al., 2016).

\section{Acknowledgements}

We are grateful to A. Hewat (ILL, Grenoble) for his assistance during the D2B experiments. JE would also like to thank the Physics and Chemistry of Materials Group (T-1) at LANL for making computing resources available.

\section{Funding information}

We acknowledge the support of the Institut Laue-Langevin (ILL) in providing the neutron research facilities used in this work. EAB and PTCF are grateful for the support from the CNPq and FUNCAP. JEMP's research is supported through the Brazilian Science Without Borders (process number 207740/2014-3) program. Financial support was given for travel by Danscatt to JEMP and HNB. JE's research used resources of the National Energy Research Scientific Computing Center, a DOE Office of Science User Facility supported by the Office of Science of the US Department of Energy under Contract No. DE-AC02-05CH11231.

\section{References}

Barthès, M., Dénoyer, F., Lorenzo, J.-E., Zaccaro, J., Robert, A., Zontone, F. \& Bordallo, H. N. (2003). Eur. Phys. J. B - Condens. Matter. 37, 375-382.
Barthès, M., Vik, A. F., Spire, A., Bordallo, H. N. \& Eckert, J. (2002). J. Phys. Chem. A, 106, 5230-5241.

Bordallo, H. N., Barthès, M. \& Eckert, J. (1997). Physica B, 241-243, 1138-1140.

Crowell, R. A. \& Chronister, E. L. (1993). Phys. Rev. B, 48, 172177.

Dalton, J. B. \& Schmidt, C. L. A. (1933). J. Biol. Chem. 103, 549-578.

Destro, R., Marsh, R. E. \& Bianchi, R. (1988). J. Phys. Chem. 92, 966973.

Destro, R., Soave, R. \& Barzaghi, M. (2008). J. Phys. Chem. B, 112, 5163-5174.

Frisch, M. J. et al. (2009). Gaussian 09, Revision A. Gaussian Inc., Wallingford, Connecticut, USA.

Ganesan, A., Brunger, M. J. \& Wang, F. (2013). Eur. Phys. J. D, 67, 229.

Hamase, K., Morikawa, A. \& Zaitsu, K. (2002). J. Chromatogr. B, 781, 73-91.

Hudson, M. R., Allis, D. G., Ouellette, W. \& Hudson, B. S. (2009). Phys. Chem. Chem. Phys. 11, 9474-9483.

Ishikawa, K., Terasawa, Y., Tanaka, M. \& Asahi, T. (2017). J. Phys. Chem. Solids, 104, 257-266.

Kapustin, E. A., Minkov, V. S., Stare, J. \& Boldyreva, E. V. (2014). Cryst. Growth Des. 14, 1851-1864.

Karakawa, S., Shimbo, K., Yamada, N., Mizukoshi, T., Miyano, H., Mita, M., Lindner, W. \& Hamase, K. (2015). J. Pharm. Biomed. Anal. 115, 123-129.

Kimura, T., Hamase, K., Miyoshi, Y., Yamamoto, R., Yasuda, K., Mita, M., Rakugi, H., Hayashi, T. \& Isaka, Y. (2016). Sci. Rep. 6, 26137.

Kolesov, B. A. \& Boldyreva, E. V. (2011). J. Raman Spectrosc. 42 , 696-705.

Kresse, G. \& Furthmüller, J. (1996). Phys. Rev. B, 54, 11169-11186.

Kržan, M., Vianello, R., Maršavelski, A., Repič, M., Zakšek, M., Kotnik, K., Fijan, E. \& Mavri, J. (2016). PLoS One, 11, e0154002.

Lagaron, J.-M. (2002). Macromol. Symp. 184, 19-36.

Larson, A. C. \& Von Dreele, R. B. (1994). Los Alamos National Laboratory Report LAUR, 86-748.

Lehmann, M. S., Koetzle, T. F. \& Hamilton, W. C. (1972). J. Am. Chem. Soc. 94, 2657-2660.

Loh, E. (1975). J. Chem. Phys. 63, 3192-3194.

Monkhorst, H. J. \& Pack, J. D. (1976). Phys. Rev. B, 13, 5188-5192.

Perdew, J. P., Burke, K. \& Ernzerhof, M. (1996). Phys. Rev. Lett. 77, 3865-3868.

Ramirez-Cuesta, A. J. (2004). Comput. Phys. Commun. 157, 226-238.

Rozenberg, M., Shoham, G., Reva, I. \& Fausto, R. (2003). Spectrochim. Acta A Mol. Biomol. Spectrosc. 59, 3253-3266.

Salam, A. (1992). Phys. Lett. B, 288, 153-160.

Souza, J. M. de, Freire, P. T. C., Argyriou, D. N., Stride, J. A., Barthès, M., Kalceff, W. \& Bordallo, H. N. (2009). Chem. Phys. Chem., 10, 3337-3343.

Souza, J. M. de, Freire, P. T. C., Bordallo, H. N. \& Argyriou, D. N. (2007). J. Phys. Chem. B, 111, 5034-5039.

Sullivan, R., Pyda, M., Pak, J., Wunderlich, B., Thompson, J. R., Pagni, R., Pan, H., Barnes, C., Schwerdtfeger, P. \& Compton, R. (2003). J. Phys. Chem. A, 107, 6674-6680.

Susi, H. \& Byler, D. M. (1980). J. Mol. Struct. 63, 1-11.

Vik, A. F., Yuzyuk, Y. I., Barthès, M. \& Sauvajol, J.-L. (2005). J. Raman Spectrosc. 36, 749-754.

Wang, W., Min, W., Bai, F., Sun, L., Yi, F., Wang, Z., Yan, C., Ni, Y. \& Zhao, Z. (2002). Tetrahedron Asymmetry, 13, 2427-2432.

Wang, C. H. \& Storms, R. D. (1971). J. Chem. Phys. 55, 3291-3299.

Wang, W., Yi, F., Ni, Y., Zhao, Z., Jin, X. \& Tang, Y. (2000). J. Biol. Phys. 26, 51-65.

Wilson, C. C., Myles, D., Ghosh, M., Johnson, L. N. \& Wang, W. (2005). New J. Chem. 29, 1318.

Zhang, F., Wang, H.-W., Tominaga, K. \& Hayashi, M. (2015). J. Phys. Chem. A, 119, 3008-3022. 


\section{IUCrJ}

ISSN 2052-2525

CHEMISTRY|CRYSTENG

Received 12 March 2018

Accepted 16 May 2018

Edited by V. T. Forsyth, Institut Laue-Langevin, France, and Keele University, UK

Keywords: parity-violation energy; enantiomers; phase transitions; amino acids.

\section{Comments on 'Hydrogen bonds in crystalline D-alanine: diffraction and spectroscopic evidence for differences between enantiomers'}

\author{
Hans-Beat Bürgi ${ }^{\mathrm{a}, \mathrm{b} *}$ and Piero Macchi ${ }^{\mathrm{a} *}$

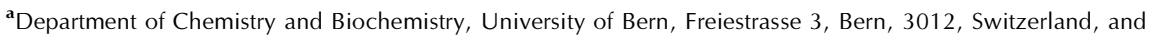 \\ ${ }^{\mathbf{b}}$ Department of Chemistry, University of Zurich, Winterthurestrasse 190, Zurich, 8057, Switzerland. *Correspondence \\ e-mail: hans-beat.buergi@krist.unibe.ch, piero.macchi@dcb.unibe.ch
}

The recent paper by Belo, Pereira, Freire, Argyriou, Eckert \& Bordallo [(2018), IUCrJ, 5, 6-12] reports observations that may lead one to think of very strong and visible consequences of the parity-violation energy difference between enantiomers of a molecule, namely alanine. If proved, this claim would have an enormous impact for research in structural chemistry. However, alternative, more realistic, explanations of their experiments have not been ruled out by the authors. Moreover, the theoretical calculations carried out to support the hypothesis are unable to differentiate between enantiomers (molecules or crystals). Therefore, the conclusions drawn by Belo et al. (2018) are deemed inappropriate as the data presented do not contain sufficient information to reach such a conclusion.

\section{Introduction}

In a recent paper, entitled 'Hydrogen bonds in crystalline D-alanine: diffraction and spectroscopic evidence for differences between enantiomers' (our emphasis), Belo et al. (2018) report polarized Raman spectra collected on hydrogenated D-alanine single crystals $\left(\mathrm{C}_{3} \mathrm{H}_{7} \mathrm{NO}_{2}, \mathrm{D}-\mathrm{ala}-\mathrm{h}_{7}\right)$, neutron powder diffraction (NPD) measurements on fully deuterated $\mathrm{D}-a \mathrm{al}^{-} \mathrm{d}_{7}$ and $a b$ initio calculations of the harmonic vibrational frequencies of an isolated D-ala molecule and of D- and L-ala crystals. In the abstract of the paper, the authors conclude that their 'results reveal dissimilarities in the structural properties of D-alanine compared with L-alanine' (our emphasis). These are very strong statements. In their generality, they are no less than a refutation of the empirically and theoretically founded principle that enantiomeric molecules have the same energies and the same chemical properties; optical activity and circular dichroism are the same in absolute value but have opposite sign.

The present doctrine derives from the Schrödinger equation with a Hamilton operator accounting for electromagnetic forces. This theory has not only been shown to explain successfully and quantitatively all kinds of experimental chemical and physical results, it is also parity invariant, i.e. its mathematical structure requires that the energies of enantiomers be identical; their equilibrium structures and their potential energy surfaces must be exact mirror images of each other and their vibrational spectra identical (Quack, 2014). Differences between enantiomeric molecules are only possible in a theory that violates parity, i.e. a theory that accounts for the weak nuclear force, the only kind of force that breaks parity (see, for example, Quack et al., 2008). Belo et al. (2018) allude to this possibility in the introduction to their report without mentioning, however, that energy differences between enantiomers due to parity violation (PV) are extremely small. For D- and L-ala, differences of the order of $10^{-14} \mathrm{~kJ} \mathrm{~mol}^{-1}$ have been calculated in both the gaseous and aqueous phases and have been found to depend on conformation, i.e. for some conformations D-ala is more stable, for others L-ala is more stable (Laerdahl et al., 2000; Berger \& Quack, 2000; Quack, 2014). On the basis of such calculations, Quack and collaborators estimated that an experimental verification of these differences by vibrational spectroscopy of suitable molecules would require a spectral resolution $\Delta v_{\mathrm{PV}} / v$ 
of the order of $10^{-16}$ to $10^{-19}$ (Quack et al., 2008), a value which has not yet been reached with present day technology (Albert et al., 2017). It is therefore highly unlikely that PV affects molecular and crystal structures as well as their energies and vibrational spectra in a way that is observable from present day diffraction and spectroscopic experiments. ${ }^{1}$

If correct, the far-reaching interpretation of their experimental data by Belo et al. (2018) represents a refutation of the basic tenet implicit in the usual quantum chemical Schrödinger equation that currently represents so-called 'normal science' according to Kuhn (1962). It could thus represent the beginning of a scientific revolution that might lead to the formulation of a new paradigm (and perhaps a revision of the currently accepted values of parity-violation energy) and, by accumulation of additional evidence, to a new 'normal science'. Given the potential consequences of such events, it is mandatory to confirm the new evidence with every imaginable and feasible control experiment and to eliminate conventional explanations of the new evidence as far as possible. We comment on the paper by Belo et al. (2018) with these thoughts in mind.

\section{Some general comments on comparing and interpreting data of enantiomers}

Our comments are guided by five main questions:

\subsection{Are all data available for both enantiomers?}

If not, any observation judged unusual cannot necessarily be attributed to a difference between enantiomers, as the same or a similar observation might be made for the opposite enantiomer as well. We note that the Raman scattering data for D-ala- $\mathrm{h}_{7}$ reported by Belo et al. (2018) are not matched with correspondingly detailed data for $\mathrm{L}-\mathrm{ala}-\mathrm{h}_{7}$. Thus, it cannot be excluded that any 'unusual' observation in one enantiomer might also be found in the other one, potentially making the two enantiomers the same.

2.2. Have alternative explanations, not related to the putative phase transition associated with the Salam hypothesis on interconversion between enantiomers, been considered for 'unusual' observations in only one enantiomer?

In the early 1990s, Salam $(1991,1992)$ suggested that parity violation may imply a second-order phase transition below a critical temperature involving tunnelling of the less stable into the more stable enantiomer. Subsequently, several authors reported observations that were interpreted as evidence supporting Salam's hypothesis (e.g. Wang et al., 2002; Belo et al., 2018) without excluding alternative explanations for their observations. Some conventional explanations of the putative unusual phenomena observed in the Raman data for D-ala- $\mathrm{h}_{7}$ are suggested below in Section 3.2.

\footnotetext{
$\mathbf{1}$ The articles by Quack reference much of the recent work by other researchers in this area.
}

2.3. If data for both enantiomers are compared, are the histories of the respective samples and their chemical analysis the same?

The powder diffraction data on D-ala-d $\mathrm{d}_{7}$ (Belo et al., 2018) and L-ala-d $\mathrm{d}_{7}$ (De Souza et al., 2009) come from two experiments published $\sim 10$ years apart. There is no comparison, neither of the histories of the two samples nor of their analytical data, e.g. the H/D ratios in the recrystallized samples. Even though deuterated water was used for the recrystallization of deuterated samples, one cannot exclude exchange of $\mathrm{D}$ for $\mathrm{H}$, especially at the $\mathrm{ND}_{3}$ group, unless the recrystallizations were carried out in a dry atmosphere. Sullivan et al. (2003) noticed that the heat of transition associated with a signal in the $C_{\mathrm{p}}$ versus $T$ specific heat curve of $\mathrm{L}-\mathrm{ala}-\mathrm{h}_{7}$ around $270 \mathrm{~K}$ decreased as the number of crystallization cycles increased. This is clear evidence for a history dependence of some sample properties. By 'history of the sample', we mean a number of features that depend on the treatment of the species before and after the preparation of crystals used for data collection (e.g. purity, degree of crystallinity, grain size, type and number of defects). The effects of such a dependence on the properties reported by Belo et al. (2018) have to be excluded before the data from two different samples can be compared conclusively.

\subsection{Have alternative explanations been considered for differences between enantiomers, i.e. explanations not related to the putative phase transition associated with the Salam hypothesis?}

Belo et al. (2018) reported significant differences between L-ala and D-ala in the positions of the $\mathrm{D}$ atoms of the ammonium groups refined from the NPD data and, consequently, different geometries of the $\mathrm{D} \cdots \mathrm{O}$ hydrogen bonds. We note that they do not report refinement of the powder data of D-ala- $\mathrm{d}_{7}$ starting from the structure model obtained from the L-ala- $\mathrm{d}_{7}$ powder data and vice versa; multiple minima in the crystallographic least-squares surface have thus not been excluded. Such an experiment would be particularly important with powder data, given their restricted information content compared with single-crystal data.

\subsection{How do the postulated differences between enantiomers} compare with the present state of quantum theory?

The periodic density functional theory (DFT) calculations of inelastic neutron scattering (INS) spectra for D- and $\mathrm{L}-\mathrm{ala}-\mathrm{h}_{7}$ discussed at the end of Section 3.1 of Belo et al. (2018) are said to show differences between L-ala and D-ala in the optimized geometries, in particular the $\mathrm{N}-\mathrm{H}$ optimized distances. In keeping with this, the calculated inelastic neutron scattering of the two enantiomeric crystals differ as well. However, Belo et al. (2018) used model Hamiltonians containing only the potentials of electromagnetic forces (GGA DFT + Vanderbilt ultra-soft pseudopotential). The weak forces that violate the parity are not included, therefore effects of PV cannot emerge from these calculations. A possible explanation for the findings of Belo et al. (2018) is suggested in Section 3.3. 


\section{Some specific comments on comparing and interpreting data from D- and L-ala}

\subsection{Data from the literature}

It is certainly true, as is also mentioned by Belo et al. (2018), that L-ala has been studied intensively as a function of temperature or pressure by both Raman spectroscopy and $\mathrm{X}$-ray and neutron diffraction. Some diffraction data sets show very high resolution and have been collected at very low temperature, as required for accurate charge-density determinations. By comparison, D-ala has been investigated much less (it is more expensive!), mainly with the intention of finding experimental confirmation of the effects of PV. While Wang et al. (2002) claimed that differences exist between the enantiomers, Sullivan et al. (2003) found no unusual behaviour in their X-ray diffraction and NMR experiments in the temperature range expected for the putative phase transition $(\sim 270 \mathrm{~K})$. They also presented arguments against the Salam hypothesis for the molecules under study. Wilson et al. (2005) could offer no structural support of the Salam hypothesis based on single-crystal neutron diffraction studies of $\mathrm{D}-\mathrm{ala}-\mathrm{h}_{7}$ and $\mathrm{L}$-ala- $\mathrm{h}_{7}$ at $60 \mathrm{~K}$ and room temperature.

Note that all single-crystal neutron diffraction experiments on both D-ala and L-ala have been performed with hydrogenated species (Wilson et al., 2005; Lehmann et al., 1972), whereas the powder diffraction data used by Belo et al. (2018) come from deuterated species, for both D- and L-ala. One might therefore be tempted to conclude that the putative PV effects occur for ala- $\mathrm{d}_{7}$ only and not for ala- $\mathrm{h}_{7}$. However, differences between hydrogenated and deuterated D- or L-ala have not been investigated with experiments of comparable accuracy, neither neutron single-crystal diffraction nor NPD for both isotopomers. This prevents a conclusive comparison between the isotopomers.

\subsection{Unusual Raman spectroscopic behaviour of one enantiomer}

The Raman studies concentrate on 'anomalies in the lattice modes of hydrogenated D-ala' (Section 3.1 of Belo et al., 2018). Two of the anomalies mentioned are the appearance of 'new bands' and 'bands that split at lower temperatures' (caption to Fig. 2 of Belo et al., 2018). One of these new bands (at $\sim 100 \mathrm{~cm}^{-1}$ in Fig. $2 a$ of Belo et al., 2018) is indicated to appear at and below $208 \mathrm{~K}$. Inspection of the figure suggests that the band is present all the way to $300 \mathrm{~K}$ as a shoulder of the very strong signal at $\sim 113 \mathrm{~cm}^{-1}$. Another such band is said to appear at $\sim 170 \mathrm{~cm}^{-1}$ below $175 \mathrm{~K}$. Both of them are identified as $B$ bands appearing in the $A$-band spectrum with small intensities. In Fig. 3( $a$ ), which shows the $B$ bands, these signals are seen at all temperatures between 21 and $290 \mathrm{~K}$. The one at $170 \mathrm{~cm}^{-1}$ shifts to lower frequency at the higher temperatures, reduces its maximal intensity and becomes broader. The behaviour of this band in the $A$ spectrum is not incompatible with its behaviour in the $B$ spectrum. Since the experimental part says nothing about the accuracy of the crystal orientation relative to the probing laser beam, it cannot be excluded that the $B$ bands in the $A$ spectrum are due to slight misorientation of the crystal. Such an explanation would make the postulated phase transition unnecessary, but is not considered.

Figs. 2(b) and 2(c) (Belo et al., 2018) are said to indicate splitting of the bands at $\sim 140 \mathrm{~cm}^{-1}$ and $\sim 138 \mathrm{~cm}^{-1}$, respectively, observed at $283 \mathrm{~K}$. The former slowly shifts position on cooling, until at $22 \mathrm{~K}$ it is found at $\sim 150 \mathrm{~cm}^{-1}$. From $160 \mathrm{~K}$ up it slowly merges with the band at $140 \mathrm{~cm}^{-1}$, which is still visible as a shoulder at $160 \mathrm{~K}$ and becomes accidentally degenerate with the shifting band at $283 \mathrm{~K}$. The band at $\sim 138 \mathrm{~cm}^{-1}$ shows similar behaviour (Fig. $2 c$ of Belo et al., 2018), with the two bands visible to at least $208 \mathrm{~K}$. These shifts indicate noticeable Grüneisen-type anharmonicity, i.e. a decrease in frequency with increasing crystal volume due to thermal expansion (Grüneisen, 1926; Kolesov, 2017). Such anharmonicity has also been deduced from the thermal evolution of atomic displacement parameters, which are mainly determined by the external lattice modes (Bürgi et al., 2000; Aree et al., 2014; the latter paper and its two predecessors discuss the closely related $\alpha$-, $\beta$ - and $\gamma$-glycine polymorphs). These observations suggest that the two bands seen at low temperatures persist all the way to room temperature, with the higher-energy band at $\sim 150 \mathrm{~cm}^{-1}$ shifting to smaller frequencies due to crystal expansion. Analogous arguments apply to the splittings discussed in Fig. 3 of Belo et al. (2018). Note that the alternative interpretation given here does not require a phase transition.

We postulate that the few examples of alternative explanations of the Raman scattering data by Belo et al. (2018) as given above - while not necessarily correct - would have had to be explicitly excluded before claiming - if only implicitly - a phase transition related to the Salam hypothesis and thus claiming 'structural dissimilarities' between enantiomers. Furthermore, a similarly detailed discussion of and comparison with corresponding data for $\mathrm{L}-\mathrm{ala}-\mathrm{h}_{7}$ is lacking.

\subsection{Comments on theoretical calculations}

Belo et al. (2018, p. 10) state 'A most noteworthy difference in the low-frequency dynamics of $\mathrm{D}$ - and L-ala is apparent when comparing the calculated $10 \mathrm{~K}$ INS spectra for L-ala versus D-ala, Fig. 5(b). It is quite obvious that there are significant differences in the vibrational amplitudes (i.e. peak intensities) of the low-frequency modes below $350 \mathrm{~cm}^{-1}$. As mentioned in Sections 1 and 2.5, the DFT calculations by Belo et al. cannot account for PV since they do not contain the corresponding operator. In the context of differences between enantiomers this evidence is meaningless.

A possible explanation of their results - one that can be tested easily - might be as follows. Starting from their neutron powder structures for D- and L-ala, Belo et al. (2018) have optimized the respective atomic positions by DFT calculations and obtained different results for D- and L-ala. There is no mention of the energy difference between the two, nor of that between the structure optimized for D-ala and the inverted DFT-optimized structure of L-ala and vice versa (nor of the transition state energy between the two optimized structures, see Sullivan et al., 2003). Could it be that the difference is a 
result of incomplete structure optimization of the different starting structures due to the convergence criteria incorporated in the DFT procedure used?

\section{Conclusion}

Based on the comments above we conclude that Belo et al. (2018) have not presented coherent and conclusive 'diffraction and spectroscopic evidence for differences between enantiomers'. Our conclusion concurs with those arrived at in earlier experimental and computational work (Berger \& Quack, 2000; Laerdahl et al., 2000; Sullivan et al., 2003; Wilson et al., 2005; Albert et al., 2017) and with the current state of quantum chemical theory, including the effects of parity violation (Quack, 2014).

In the list below, we suggest some alternative explanations for the reported differences between D-ala and L-ala $[(a)-(c)]$, and tests to confirm or exclude them $[(d),(e)]$ :

(a) Incongruent crystallization processes for the enantiomeric substances, possibly leading to differences in sample characteristics, specifically the degree of deuteration or the density of crystal defects. Differences in sample treatment have been shown to explain differences observed in scanning temperature experiments (Sullivan et al., 2003).

(b) Anharmonicity and isotope effects.

(c) Inconsistent structural optimization by the PV-free quantum chemical DFT method used.

(d) Comparison of Raman data for D- and L-ala.

(e) Tests for multiple structural minima during the refinement of NPD data.

We do not deny that the differences observed by Belo et al. (2018) are real. However, whatever they are, they have to be tested as suggested above before they can be attributed to 'differences between enantiomers'.

Note added in proof: After submission of this work we became aware of similar work on L-nucleic acids ['First look at RNA in L-configuration' (Vallazza et al., 2004) and 'First experimental evidence for the preferential stabilization of the natural $\mathrm{D}$ - over the non-natural L-configuration in nucleic acids' (Bolik et al., 2007)]. The comments given above on the interpretation of differences between experimental data on enantiomers apply a fortiori to this work. Enantiomeric biomolecules such as duplex RNA octamers are even more difficult to characterize and compare than the relatively simple ala crystals.

\section{Acknowledgements}

The authors thank Professors M. Spackman, D. Jayatilaka, G. Chandler and B. B. Iversen for careful reading of this manuscript.

\section{References}

Albert, S., Keppler, K., Boudon, V., Lerch, P. \& Quack, M. (2017). J. Mol. Spectrosc. 337, 105-123.

Aree, T., Bürgi, H.-B., Chernyshov, D. \& Törnroos, K. W. (2014). J. Phys. Chem. A, 118, 9951-9959.

Belo, E. A., Pereira, J. E. M., Freire, P. T. C., Argyriou, D. N., Eckert, J. \& Bordallo, H. N. (2018). IUCrJ, 5, 6-12.

Berger, R. \& Quack, M. (2000). ChemPhysChem, 1, 57-60.

Bolik, S., Rübhausen, M., Binder, S., Schulz, B., Perbandt, M., Genov, N., Erdmann, V., Klussmann, S. \& Betzel, Ch. (2007). RNA, 13, 1877-1880.

Bürgi, H. B., Capelli, S. C. \& Birkedal, H. (2000). Acta Cryst. A56, 425-435.

Grüneisen, E. (1926). Handbuch der Physik, edited by H. Geiger and K. Scheel, Vol. 10, pp. 1-59. Berlin: Springer.

Kolesov, B. A. (2017). J. Raman Spectrosc. 48, 323-326.

Kuhn, T. S. (1962). The Structure of Scientific Revolutions, 1st ed. University of Chicago Press.

Laerdahl, J. K., Wesendrup, R. \& Schwerdtfeger, P. (2000). ChemPhysChem, 1, 60-62.

Lehmann, M. S., Koetzle, T. F. \& Hamilton, W. C. (1972). J. Am. Chem. Soc. 94, 2657-2660.

Quack, M. (2014). Eur. Rev. 22, S50-S86.

Quack, M., Stohner, J. \& Willeke, M. (2008). Annu. Rev. Phys. Chem. 59, 741-769.

Salam, A. (1991). J. Mol. Evol. 33, 105-113.

Salam, A. (1992). Phys. Lett. B, 288, 153-160.

Souza, J. M. De, Freire, P. T. C., Argyriou, D. N., Stride, J. A., Barthès, M., Kalceff, W. \& Bordallo, H. N. (2009). ChemPhysChem, 10, 3337-3343.

Sullivan, R., Pyda, M., Pak, J., Wunderlich, B., Thompson, J. R., Pagni, R., Pan, H., Barnes, C., Schwerdtfeger, P. \& Compton, R. (2003). J. Phys. Chem. A, 107, 6674-6680.

Vallazza, M., Perbandt, M., Klussmann, S., Rypniewski, W., Einspahr, H. M., Erdmann, V. A. \& Betzel, Ch. (2004). Acta Cryst. D60, 1-7. Wang, W., Min, W., Bai, F., Sun, L., Yi, F., Wang, Z., Yan, C., Ni, Y. \& Zhao, Z. (2002). Tetrahedron Asymmetry, 13, 2427-2432.

Wilson, C. C., Myles, D., Ghosh, M., Johnson, L. N. \& Wang, W. (2005). New J. Chem. 29, 1318-1322. 